\title{
CONSTRUCTING LOCALLY CONNECTED NON-COMPUTABLE JULIA SETS
}

\author{
MARK BRAVERMAN, MICHAEL YAMPOLSKY
}

\begin{abstract}
A locally connected quadratic Siegel Julia set has a simple explicit topological model. Such a set is computable if there exists an algorithm to draw it on a computer screen with an arbitrary resolution. We constructively produce parameter values for Siegel quadratics for which the Julia sets are non-computable, yet locally connected.
\end{abstract}

\section{Preliminaries}

In this paper, we will assume that the reader is familiar with the concept of computability of a subset of $\mathbb{R}^{n}$ and its applications to Julia sets of rational functions. We refer the reader to our paper [BY08a] and the book [BY08b] for an introduction to computability of functions and sets in $\mathbb{R}^{n}$, as it applies to the study of Julia sets. A detailed treatment of computability over the reals is found in [Wei00].

We will denote

$$
f_{c}(z)=z^{2}+c, \text { and } P_{\theta}(z)=z^{2}+e^{2 \pi i \theta} z
$$

two parameterizations of the quadratic family. The latter is more convenient in studying quadratics with a neutral fixed point. We denote $J_{c}, J_{\theta}$ and $K_{c}, K_{\theta}$ the Julia sets and the filled Julia sets respectively. Suppose, a polynomial $f_{c}$ has a periodic Siegel disk $\Delta$ centered at a point $\zeta$. Consider a conformal isomorphism $\phi: \mathbb{D} \mapsto \Delta$ mapping 0 to $\zeta$. The conformal radius of the Siegel disk $\Delta$ is the quantity

$$
r(\Delta)=\left|\phi^{\prime}(0)\right|
$$

A polynomial $P_{\theta}$ with $\theta \in \mathbb{R}$ has a neutral fixed point at the origin. When this point is of Siegel type, we denote $\Delta_{\theta}$ the Siegel disk around it, and set

$$
r(\theta)=r\left(\Delta_{\theta}\right)
$$

For all other values of $\theta \in \mathbb{R}$ we set $r(\theta)=0$.

Informally, the Julia set $J_{c}$ (or $J_{\theta}$ ) is computable if, given arbitrarily good approximations of the parameter c (or $\theta$ ), a Turing Machine can output images of $J_{c}$ (or $J_{\theta}$ ) with an arbitrarily high resolution. The parameter is provided to the machine via an oracle, which the machine can query with an arbitrarily high precision. In [BY06] we showed that, surprisingly, there exist parameters $c$ for which the Julia set $J_{c}$ is not computable. In [BY08a] we demonstrated that such parameters can themselves be computed with an arbitrary precision by an explicit algorithm. The practical implications of these results are quite striking: there are computable values of $c$ for which $J_{c}$ cannot be visualized numerically.

Date: October 7, 2008.

This research was partially conducted during the period the first author was employed by the Clay Mathematics Institute as a Liftoff Fellow.

The second author's research is supported by NSERC operating grant. 
As we showed in [BBY07], a quadratic polynomial with an uncomputable Julia set necessarily possesses a cycle of Siegel disks. Further, we demonstrated in [BBY07]:

Theorem 1.1. The set $J_{c}$ is non-computable if and only if it possesses a periodic Siegel disk $\Delta$, whose conformal radius $r(\Delta)$ cannot be computed with an oracle access to the value of $c$.

For a parameter $\theta \in \mathbb{R}$, the Julia set $J_{\theta}$ is computable by a Turing Machine with an oracle for $\theta$ if and only if the number $r(\theta)$ is computable with an oracle for $\theta$.

A number $r \in \mathbb{R}$ is called right-computable if there exists a Turing Machine which computes a non-decreasing sequence of rationals

$$
r_{n} \searrow r
$$

A left-computable real is defined in a similar way, replacing a non-decreasing sequence with a non-increasing one. The set of right computable reals is larger than the set of computable reals (in fact, a real number is computable if and only if it is simultaneously right- and left-computable). The precise form of the main result of our paper [BY08a] is:

Theorem 1.2. Let a real number

$$
r \in\left(0, \sup _{\theta \in \mathbb{T}} r(\theta)\right)
$$

Then $r=r(\theta)$ is the conformal radius of a Siegel disk $\Delta_{\theta}$ with a computable parameter $\theta$ if and only if $r$ is right-computable.

By Theorem 1.1, any right-computable number that is not computable gives rise to a noncomputable Julia set with a computable parameter $\theta$.

It is natural to expect that the picture of a Julia set which cannot be effectively visualized is topologically complicated. As we have shown in [BY06], the topology of some such sets is indeed pathological. However, in [BY08b] (Theorem 6.16) we showed that there exist parameters $\theta$ for which the Julia set $J_{\theta}$ is non-computable and locally connected. Such sets have simple and explicit topological models. Our proof was non-constructive in an essential way, so it was not clear whether a value $\theta$ with these properties might be computable.

In this paper we answer in the affirmative:

Main Theorem. There exists a computable value of $\theta$ for which the Julia set $J_{\theta}$ is noncomputable and locally connected.

Thus the picture of $J_{\theta}$ is "nice" (has an explicit and simple topological model), the parameter is "nice" (computable), and yet no algorithm to draw $J_{\theta}$ exists. The proof of the Main Theorem will require both sophisticated tools of Complex Dynamics and Renormalization Theory, and a new approach to constructing a non-computable Julia set. We begin by outlining useful facts about locally connected Siegel Julia sets in the next section.

\section{LoCALLY CONNECTED QUADRATIC JUlia SETS}

2.1. Local connectedness of sets in $\mathbb{C}$. Recall, that a topological space $X$ is locally connected if for each point $x \in X$ there exists a sequence of neighborhoods $U_{i}(x) \ni x$ such that:

(1) $U_{i}(x)$ is open and connected in $X$;

(2) $\cap U_{i}(x)=\{x\}$. 
We remark that the condition (1) can be weakened:

(1a) $U_{i}(x)$ is connected in $X$ and contains an open neighborhood around $x$.

The main significance of local connectedness in the study of quadratic Julia sets comes from the following construction. Consider a quadratic polynomial $f_{c}(z)=z^{2}+c$ with a connected Julia set. The Riemann mapping

$$
\Phi: \hat{\mathbb{C}} \backslash K_{c} \rightarrow \hat{\mathbb{C}} \backslash \overline{\mathbb{U}}
$$

is uniquely determined by the normalization $\Phi(\infty)=\infty$ and $\Phi(z) \sim z$ for $z \rightarrow \infty$. It then coincides with the Bötcher coordinate of $f_{c}(z)$ at infinity:

$$
\Phi\left(f_{c}(z)\right)=(\Phi(z))^{2} .
$$

As the map $z \mapsto z^{2}$ preserves the polar coordinate grid on $\hat{\mathbb{C}} \backslash \overline{\mathbb{U}}$, the equation $(2.1)$ implies that the preimages of polar coordinate lines under $\Phi$ form an invariant grid for $f_{c}$. In particular, each radial curve

$$
R_{\theta} \equiv \Phi^{-1}\left(\left\{r e^{2 \pi i \theta} \mid r \in(1, \infty)\right\}\right)
$$

is mapped onto the curve $R_{\theta^{\prime}}$ by $f_{c}$, with $\theta^{\prime} \equiv 2 \theta \bmod \mathbb{Z}$. These curves are known as the external rays of $J_{c}$. For a fixed angle $\theta$, as $r \rightarrow 1+$, the points $r e^{2 \pi i \theta}$ approach the Julia set $J_{c}$. We say that a ray $R_{\theta}$ lands at a point $z \in J_{c}$ if

$$
\lim _{r \rightarrow 1+} \Phi^{-1}\left(r e^{2 \pi i \theta}\right)=z .
$$

In this case, the point $z$ is accessible from infinity.

The equipotential curve $E_{r}$ for $r>0$ is the preimage

$$
E_{r} \equiv \Phi^{-1}\left(\left\{e^{2 \pi i \theta \cdot r} \mid \theta \in \mathbb{T}\right\}\right) .
$$

It is mapped to $E_{2 r}$ by $f_{c}$.

It is well-known that a connected Julia set may fail to be locally connected. In particular, the following theorem was proved by Doaudy and Sullivan [Sul83], and independently by Lyubich [Lyu86]:

Proposition 2.1. If a polynomial $f_{c}$ has a periodic point of Cremer type, then its Julia set is not locally connected. Moreover, if $f_{c}$ has a cycle of Siegel disks, and $J_{c}$ is locally connected, then necessarily the critical point 0 of $f_{c}$ is contained in the boundary of one of the periodic Siegel disks.

In the case when the Julia set $J_{c}$ is locally connected, a key to its topological structure is given by the Theorem of Carathéodory. Recall that a set $K \subset \mathbb{C}$ is full if its complement is connected in $\mathbb{C}$ :

Caratheodory's Theorem. For a connected compact and full set $K \subset \mathbb{C}$ denote $\Phi$ the Riemann mapping

$$
\Phi: \hat{\mathbb{C}} \backslash K \rightarrow \hat{\mathbb{C}} \backslash \overline{\mathbb{U}} \text { with } \Phi(\infty)=\infty \text { and } \Phi^{\prime}(\infty)=1
$$

Then the following conditions are equivalent:

- the set $K$ is locally connected;

- the set $J=\partial K$ is locally connected;

- the inverse mapping $\Phi^{-1}$ extends continuously to a map $S^{1} \rightarrow J$;

- every radial ray $\Phi^{-1}\left(\left\{r e^{2 \pi i \theta} \mid r>1\right\}\right)$ lands at a point of $J$. 
As an immediate corollary we have the following:

Corollary 2.2. Assume that the Julia set of $f_{c}$ is connected and locally connected. Then the inverse Böttcher map $\Phi^{-1}$ continuously extends to a surjection $\psi: S^{1} \rightarrow J_{c}$ which is a semi-conjugacy

$$
\psi\left(z^{2}\right)=f_{c}(\psi(z))
$$

The parametrization

$$
\gamma_{c}: \theta \mapsto z=\exp (2 \pi i \theta) \mapsto \psi(z) \in J_{c}
$$

is known as the Carathéodory loop of $J_{c}$.

2.2. Locally connected Siegel Julia sets. As was first shown by Herman [Her85], there exist quadratic polynomials in the family $P_{\theta}(z)=z^{2}+e^{2 \pi i \theta} z$ with a Siegel disk $\Delta_{\theta}$ at the origin, such that the critical point

$$
p_{\theta}=-e^{2 \pi i \theta} / 2 \notin \partial \Delta_{\theta} .
$$

By Proposition 2.1 in this case $J_{c}$ is not locally connected.

There exist, however, topologically well-behaved examples with Siegel disks as we will see in the following section.

Assume now that $P_{\theta}$ has a Siegel disk with the critical point $p_{\theta}=-e^{2 \pi i \theta} / 2$ in the boundary. Assume further that this point is accessible from infinity. In this case, $J_{\theta} \backslash\left\{p_{\theta}\right\}$ has two connected components; we denote $L_{0}$ the one which does not contain $\Delta_{\theta}$. A limb of generation $n$ is a component of $P_{\theta}^{-n}\left(L_{0}\right)$.

There exist various natural ways of labeling limbs of generation $n$. For instance, denote $R_{1}$ and $R_{2}$ the two external rays which land at $p_{\theta}$, and set

$$
\Gamma=R_{1} \cup R_{2} \cup\left\{p_{\theta}\right\} .
$$

Then we have two well-defined branches of the inverse map $P_{\theta}^{-1}$ mapping $\mathbb{C} \backslash P_{\theta}(\Gamma)$ to one of the components of $\mathbb{C} \backslash \Gamma$. Let us denote $\psi_{0}$ the inverse branch which fixes $\Delta_{\theta}$, and $\psi_{1}$ the other one. We can then distinguish the limbs of the same generation by the order in which the two inverse branches were applied, so for $\bar{\sigma} \in\{0,1\}^{n}$, we have

$$
L_{\bar{\sigma}}=\psi_{\sigma_{n}} \circ \cdots \circ \psi_{\sigma_{1}}\left(L_{0}\right) \text {. }
$$

Theorem 2.3. The Julia set $J_{\theta}$ is locally connected if and only if the following three properties hold:

(I) $\partial \Delta_{\theta}$ is a Jordan curve, and contains $p_{\theta}$;

(II) the point $p_{\theta}$ is accessible from infinity;

(III) there exists a positive function $s: \mathbb{N} \rightarrow \mathbb{R}$ with $s(n) \underset{n \rightarrow \infty}{\longrightarrow} 0$ such that the diameter of each limb of generation $n$ is bounded from above by $s(n)$.

The necessity of the condition (III) is not difficult to see. If there existed a non-trivial accumulation set of an infinite sequence of limbs (a "ghost limb") then all its points would have to correspond to a single external ray $R_{\theta}$, in violation of Carathéodory's Theorem.

As for the sufficiency of conditions (I)-(III), the limbs themselves can be used to construct a basis of connected neighborhoods. For more details, see e.g. [Yam99].

Note that, if $J_{\theta}$ is locally connected, then by Theorem 2.3 (II) and Carathéodory's Theorem the conformal linearizing coordinate

$$
\phi_{\theta}: \underset{4}{\mathbb{U}} \rightarrow \Delta_{\theta}
$$



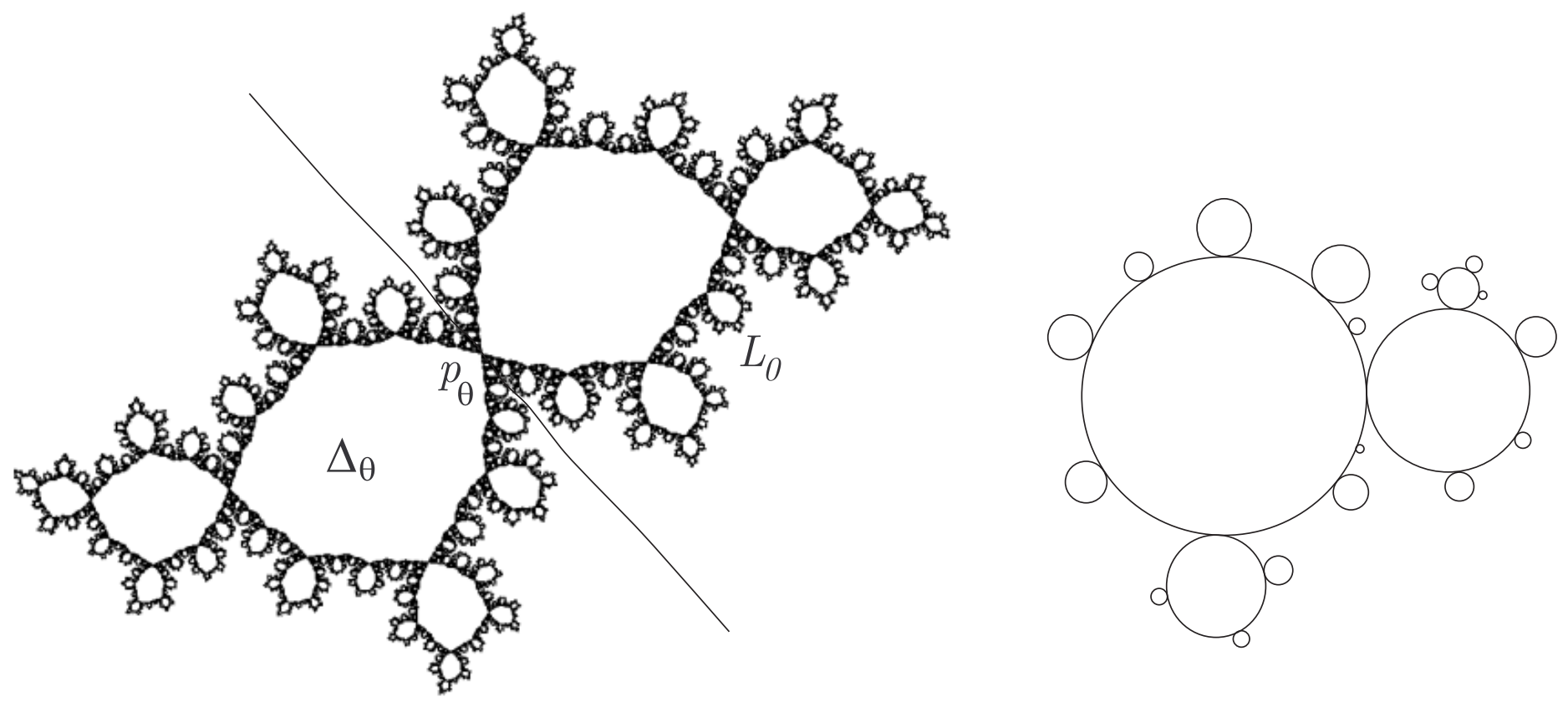

Figure 1. On the left is the filled Julia set of the quadratic polynomial $P_{\theta}$ with $\theta=\frac{1}{1+\frac{1}{1+\cdots}}$. The critical point $p_{\theta}$ is on the boundary of the Siegel disk; the two external rays landing at $p_{\theta}$ and the initial limb $L_{0}$ are also indicated. On the right is an illustration of the topological model of $J_{\theta}$.

extends continuously to the boundary. Hence the restriction

$$
P_{\theta}: \partial \Delta_{\theta} \rightarrow \partial \Delta_{\theta}
$$

is conjugated by a homeomorphic change of coordinates $\phi_{\theta}: S^{1} \rightarrow \partial \Delta_{\theta}$ to an irrational rotation of the circle. As $p_{\theta} \in \partial \Delta_{\theta}$, we obtain the following:

Proposition 2.4. If $J_{\theta}$ is locally connected, then

$$
\partial \Delta_{\theta}=\overline{\operatorname{Postcrit}\left(P_{\theta}\right)}
$$

If $J_{\theta}$ is locally connected, then a topological model for the dynamics of $P_{\theta}: J_{\theta} \rightarrow J_{\theta}$ can be constructed similarly to what is done in [Thu] and [Dou93]. However, if we are interested in constructing a topological model of $J_{\theta}$ without the dynamics, the exercise becomes rather trivial. We can, for instance, replace the Siegel disk itself, as well as its every preimage, with a round circle. Each of the circles has a countable set of circles attached to its boundary, at a dense set of points. Putting them together has to be done so that there are no intersections not only of the circles themselves, but of the closures of infinite chains of circles. 


\section{Examples of computable and locally connected Siegel Julia sets: PARAMETERS OF BOUNDED TYPE}

An irrational angle $\theta$ is said to be of type bounded by $B$ if it is represented by an infinite continued fraction with positive terms

$$
\theta=\left[a_{0}, a_{1}, a_{2}, \ldots\right] \text { such that } \sup a_{i} \leq B .
$$

The union of all numbers of a bounded type are Diophantine numbers of exponent 2; a zero measure subset of $\mathbb{T}$. As we have shown in [BBY07]:

Theorem 3.1. If $\theta$ is of a bounded type, then $r(\theta)$ is a computable real. Moreover, all such $r(\theta)$ can be computed by a single algorithm with a single parameter - an upper bound $B$ on the coefficients of the continued fraction of $r(\theta)$.

To outline the proof of this below, we recall that Siegel quadratic Julia of bounded type sets may be constructed by means of quasiconformal surgery (cf. [Dou88]) on a Blaschke product

$$
f_{\gamma}(z)=e^{2 \pi i \tau(\gamma)} z^{2} \frac{z-3}{1-3 z}
$$

This map homeomorphically maps the unit circle $\mathbb{T}$ onto itself with a single (cubic) critical point at 1 . The angle $\tau(\gamma)$ can be uniquely selected in such a way that the rotation number of the restriction $\rho\left(\left.f_{\gamma}\right|_{\mathbb{T}}\right)=\gamma$.

For each $n$, the points

$$
\left\{1, f_{\gamma}(1), f_{\gamma}^{2}(1), \ldots, f_{\gamma}^{q_{n+1}-1}(1)\right\}
$$

form the $n$-th dynamical partition of the unit circle. The following result is due to Swiatek and Herman (for the proof see e.g. Theorem 3.1 of [dFdM99]):

Theorem 3.2 (Universal real a priori bound). There exists an explicit constant $B>1$ independent of $\gamma$ and $n$ such that the following holds. Let $\gamma \in \mathbb{R} \backslash \mathbb{Q}$ and $n \in \mathbb{N}$. Then any two adjacent intervals $I$ and $J$ of the $n$-th dynamical partition of $f_{\gamma}$ are $B$-commensurable:

$$
B^{-1}|I| \leq|J| \leq B|I|
$$

Proposition 3.3 ([Her86]). For each bounded type $\gamma=\left[a_{0}, \ldots, a_{k}, \ldots\right]$ the Blaschke product $f_{\gamma}$ is $K_{1}$-quasisymmetrically conjugate to the rotation $R_{\gamma}: x \mapsto x+\gamma \bmod \mathbb{Z}$. The quasisymmetric constant may be taken as $K_{1}=\left(2 \sup a_{i}\right)^{10 B^{2}}$.

Let us now consider the mapping $\Psi$ which identifies the critical orbits of $f_{\gamma}$ and $P_{\gamma}$ by

$$
\Psi: f_{\gamma}^{i}(1) \mapsto P_{\gamma}^{i}\left(c_{\gamma}\right)
$$

We have the following (see, for example, Theorem 3.10 of [YZ01]):

Theorem 3.4 (Douady, Ghys, Herman, Shishikura). The mapping $\Psi$ extends to a $K$-quasiconformal homeomorphism of the plane $\mathbb{C}$ which maps the unit disk $\mathbb{D}$ onto the Siegel disk $\Delta_{\gamma}$. The constant $K$ may be taken as the quasiconformal dilatation of any global quasiconformal extension of the $K_{1}$-qs conjugacy of Proposition 3.3. In particular, $K \leq 2 K_{1}$.

Elementary combinatorics implies that each interval of the $n$-th dynamical partition contains at least two intervals of the $(n+2)$-nd dynamical partition. This in conjunction with 
Theorem 3.2 implies that the size of an interval of the $(n+2)$-nd dynamical partition of $f_{\gamma}$ is at most $\tau^{n}$ where

$$
\tau=\sqrt{\frac{B}{B+1}} .
$$

Hence, setting

$$
\Omega_{n}=\left\{P_{\gamma}^{i}\left(c_{\gamma}\right), i=0, \ldots, q_{n+2}\right\}
$$

by Theorem 3.4,

$$
\operatorname{dist}_{H}\left(\Omega_{n}, \partial \Delta_{\gamma}\right)<K \tau^{n}
$$

We quote:

Lemma 3.5 (see [BY08a]). Let $U$ be a simply-connected bounded subdomain of $\mathbb{C}$ containing the point 0 in the interior. Suppose $V \subset U$ is a simply-connected subdomain of $U$, and $\partial V \subset B(\partial U, \epsilon)$. Let $r(U, 0)$ denote the conformal radius of $U$ with respect to 0 , and similarly for $r(V, 0)$. Then

$$
r(U, 0)-r(V, 0) \leq 4 \sqrt{r(U, 0)} \sqrt{\epsilon} .
$$

Moreover, denote $F(x)=4 x /(1+x)^{2}$. Then

$$
r(V, 0) \leq r(U, 0) F\left(\frac{\rho(V, 0)}{\rho(U, 0)}\right) .
$$

In combination with (3.1), the above lemma yields an algorithm for computing the value of $r(\theta)$ with an arbitrary desired precision. This finishes the sketch of proof of Theorem 3.1.

The existence of locally connected Siegel Julia sets was first demonstrated by Petersen [Pet96]. A different proof was also given by the second author in [Yam99]:

Theorem 3.6 ([Pet96]). If $\theta$ is an irrational number of bounded type, then the Julia set $J_{\theta}$ is locally connected.

A work of Petersen and Zakeri [PZ04] later extended this result to a class of rotation numbers $\theta$ having full measure in $S^{1}$.

\section{Control of the postcritical set of a Siegel quadratic}

4.1. Cylinder renormalization. Cylinder renormalization is the tool which we will use to gain control of the postcritical set of $P_{\theta_{n}}$ in the above discussion. It was introduced by the second author in [Yam02], and applied to maps with Siegel disks in [Yam08]. We refer the reader to these two works for a more detailed description.

To define the procedure, we start with an analytic map $f$ defined in a neighborhood $W$ of the origin, and of the form

$$
f(z)=e^{2 \pi i \theta} z+o(z)
$$

where $\theta$ is some Brjuno number. Recall that $\left\{p_{n} / q_{n}\right\}$ denote its rational convergents. Fix some $n \geq 0$. Assume that there exists a simple arc $l \subset W$ which connects a fixed point $a$ of the iterate $f^{q_{n}}$ to 0 , and has the property that $f^{q_{n}}(l)$ is again a simple arc whose only intersection with $l$ is at the two endpoints. Let $C_{f}$ be the topological disk in $\mathbb{C} \backslash\{0\}$ bounded by $l$ and $f^{q_{n}}(l)$. We say that $C_{f}$ is a fundamental crescent if the inverse branch $\left.f^{-q_{n}}\right|_{C_{f}}$ mapping $f^{q_{n}}(l)$ to $l$ is defined and univalent, and the quotient of

$$
\overline{C_{f} \cup f^{-q_{n}}\left(C_{f}\right)} \backslash\{0, a\}
$$


by the iterate $f^{q_{n}}$ is conformally isomorphic to $\mathbb{C} / \mathbb{Z}$.

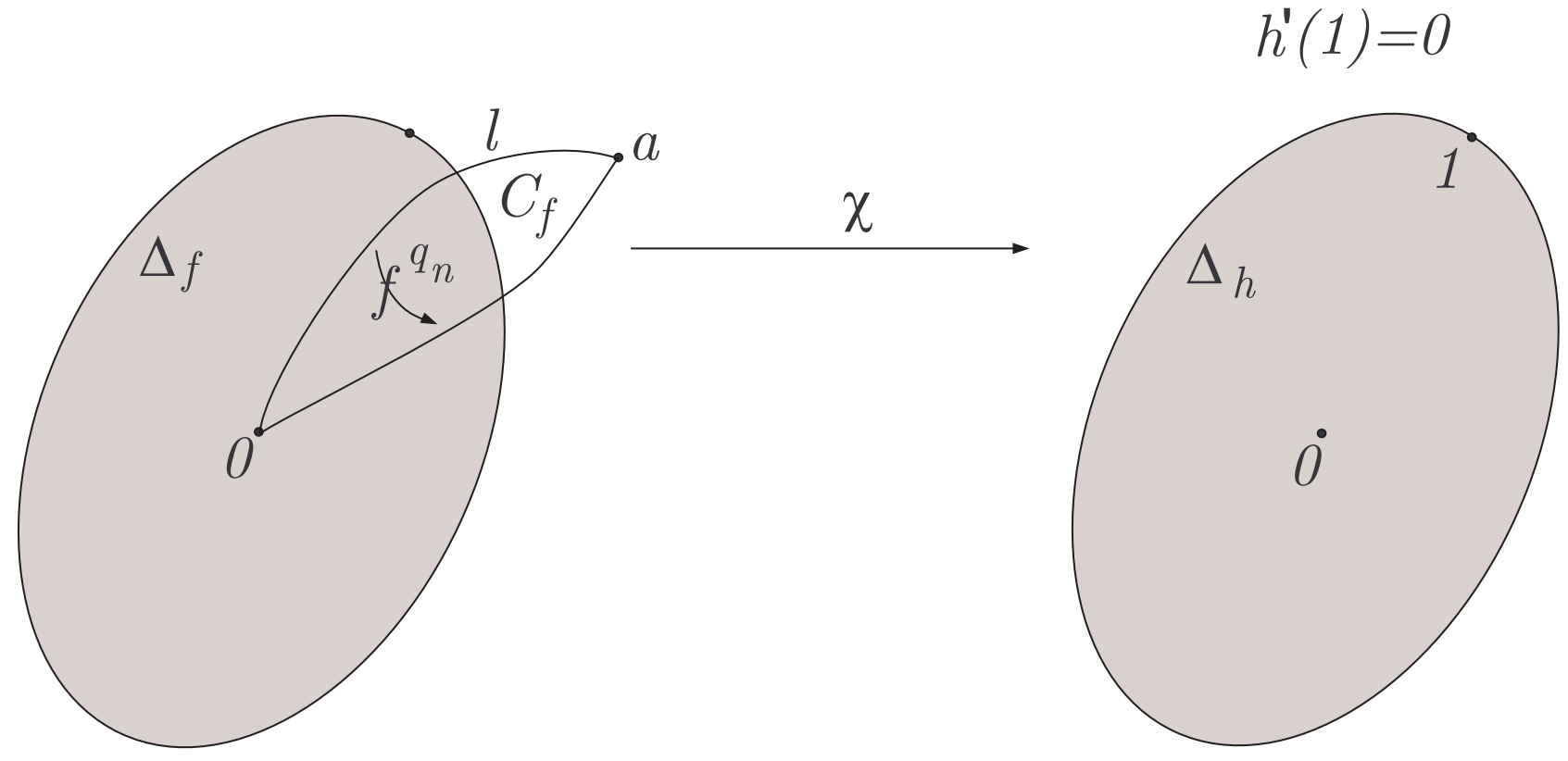

FiguRE 2. Schematics of cylinder renormalization

For a point $z$ in the fundamental crescent, consider the first return map $R_{f}(z)$ given by the smallest iterate $f^{i}(z)$ which is again contained in $C_{f}$, assuming such an $i$ exists. It will, of course, exist, and will be locally constant for all $z$ in the intersection of $C_{f}$ with the Siegel disk $\Delta_{f}$.

Let us now select a conformal isomorphism

$$
\kappa:\left(\overline{C_{f} \cup f^{-q_{n}}\left(C_{f}\right)} \backslash\{0, a\}\right) / f^{q_{n}} \stackrel{\simeq}{\longrightarrow} \mathbb{C} / \mathbb{Z},
$$

which sends the puncture at $\{0\}$ to the "upper" end $+i \cdot \infty$ of $\mathbb{C} / \mathbb{Z}$. Its composition with the exponential map $\chi(z)=\exp (2 \pi i \kappa(z))$ maps the quotient of the crescent to the complex plane punctured at the origin. Consider the map

$$
h=\chi \circ R_{f} \circ \chi^{-1}
$$

It is not difficult to see that it is an analytic function defined in a neighborhood of the origin. Moreover, filling in the removable singularity at 0 , we have:

$$
h=\exp \left(2 \pi i \theta^{\prime}\right) z+o(z), \text { with } \theta^{\prime}=G^{n+1}(\theta),
$$

where $G(\theta)=\left\{\frac{1}{\theta}\right\}$ is the Gauss map. How well-defined is $h$ ? First, and most crucially, the Liouville's Theorem implies that the only flexibility we have in the choice of $\chi$ is in postcomposing it with a homothety around 0 . A different choice of $C_{f}$ could, a priori produce a different $h$. However, 
Proposition 4.1. Every other fundamental crescent $C_{f}^{\prime}$ with the same endpoints as $C_{f}$, and such that $C_{f}^{\prime} \cup C_{f}$ is a topological disk, produces the same renormalized map $h$ (defined up to a change of coordinates by a homothety).

Now, let us suppose that $\theta$ is of bounded type, and the Siegel disk $\Delta_{f}$ is contained in the domain $W$ of $f$. Further, let the boundary of $\Delta_{f}$ contain a unique critical point of $f$. Then $h$ is also going to have a single critical point on the boundary of its Siegel disk. Let us uniquely specify $\chi$ by putting this point at 1 . We then call the map $h$ a cylinder renormalization of $f$ with period $q_{n}$.

The boundary of the Siegel disk of $h$ is obtained by a conformal "blow-up" of an arc of the boundary of $\Delta_{f}$. The cylinder renormalization acts as a zoom-in into the postcritical set.

Now let us specialize to the case of quadratic polynomials $P_{\theta}$ of a bounded type:

Theorem 4.2. Let $\theta$ be of a bounded type. Let $P_{\theta}$ be as above. There exists a sequence $g_{n}$, $n \in \mathbb{N}$ of cylinder renormalizations of $P_{\theta}$ with the following properties.

(I) For each $n$, the map $g_{n}$ is a cylinder renormalization of $P_{\theta}$ with period $q_{n}$. Thus $g_{n}$ has a Siegel disk with rotation number $G^{n+1}(\theta)$ centered at the origin, whose boundary is a quasicircle, containing the critical point 1.

(II) Denoting $C_{n}$ the fundamental crescent of the respective renormalization, we have

$$
d_{n}=\sup _{z \in C_{n}} \operatorname{dist}\left(z, \Delta_{\theta}\right) \rightarrow 0 .
$$

Moreover, $d_{n}$ is $K$-commensurable with $C_{n} \cap \partial \Delta_{\theta}$ for a universal $K>0$. Hence

$$
d_{n}<A b^{-n} \text { for some } A>0 \text {, and } b>1 \text {. }
$$

(III) Finally, there exists $k \in \mathbb{N}$ such that for all $n_{1}$ and for $n_{2} \geq n_{1}+k$, the map $g_{n_{2}}$ is a cylinder renormalization of $g_{n_{1}}$.

What can we say about the sequence of the cylinder renormalizations thus obtained? A recent result of Inou and Shishikura [IS07] implies that under an additional assumption on $\theta$ all of these analytic maps belong to a compact family:

Theorem 4.3. There exist $N_{0} \in \mathbb{N}$, a pair of topological disks $\widetilde{W} \ni W \ni\{0,1\}$, an open set $\mathcal{V}$ in the Banach space of analytic maps in $W$ with the sup-norm, and a compact subset $\mathcal{Y} \Subset \mathcal{V}$ such that the following is true.

- Let $\theta=\left[a_{1}, a_{2}, \ldots\right] \in(0,1) \backslash \mathbb{Q}$ with $a_{i} \geq N_{0}$. For every $f \in \mathcal{V}$ with $f^{\prime}(0)=e^{2 \pi i \theta}$ we have the following. The map $f$ is cylinder renormalizable with period $1=q_{0}$, and the corresponding cylinder renormalization

$$
g(z)=\exp (2 \pi i G(\theta)) z+o(z) \in \mathcal{Y} .
$$

Moreover, $g$ analytically extends to the larger domain $\widetilde{W}$.

- Further, consider the quadratic polynomial $f=P_{\theta}(z)$. Set $g_{n}$ to be the sequence of cylinder renormalizations of $f$ as in Theorem 4.2. Then there exists $j \in \mathbb{N}$ such that $\left.g_{j}\right|_{W} \in \mathcal{Y}$.

As an easy corollary, note that: 
Corollary 4.4. Let $g(z)$ and $W$ be as in the above theorem. Then the critical orbit

$$
\bigcup_{n \geq 0} g^{n}(1) \subset W .
$$

Proof. Indeed, the theorem implies that there exists an infinite sequence of cylinder renormalizations of the restriction $\left.g\right|_{W}$. Hence, iterates $\left(\left.g\right|_{W}\right)^{n}(1)$ are defined for arbitrarily large values of $n$.

\section{Modifying the Conformal Radius of A Siegel Disk}

Let us recall, that for an irrational $\theta \in \mathbb{T}$ the Yoccoz's Brjuno function $\Phi(\theta)$ is defined as follows. Inductively set $\theta_{1}=\theta$ and $\theta_{n+1}=\left\{1 / \theta_{n}\right\}$. In this way,

$$
\theta_{n}=\left[r_{n}, r_{n+1}, r_{n+2}, \ldots\right] \text {. }
$$

Then,

$$
\Phi(\theta)=\sum_{n=1}^{\infty} \theta_{1} \theta_{2} \cdots \theta_{n} \log \frac{1}{\theta_{n+1}} .
$$

Yoccoz [Yoc95] has shown that the sum

$$
\Phi(\theta)+\log r(\theta)
$$

is bounded from below independently of $\theta$. Buff and Chéritat [BC06] have greatly improved this result by showing that:

Theorem 5.1 ([BC06]). The function

$$
v: \theta \mapsto \Phi(\theta)+\log r(\theta)
$$

extends to $\mathbb{R}$ as a 1-periodic continuous function.

It is conjectured that:

Conjecture 5.2. The function $v$ defined by (5.1) is computable.

We note that Marmi, Moussa, and Yoccoz [MMY97] have conjectured that $v$ is Hölder with exponent $1 / 2$. This is known to be stronger than Conjecture 5.2 (see [BY08b] for details).

We will require the following technical lemma (for a proof see [BY08a] or [BY08b]).

Lemma 5.3. Fix $N \in \mathbb{N}$. For any given initial segment $I=\left[a_{0}, a_{1}, \ldots, a_{n}\right]$ and $m_{0}>0$, write $\omega=\left[a_{0}, a_{1}, \ldots, a_{n}, N, N, N, \ldots\right]$. Then for any $\varepsilon>0$, we can uniformly compute $m>m_{0}$, an integer $t$ and an integer $M$ such that if we write $\beta=\left[a_{0}, a_{1}, \ldots, a_{n}, N, N, \ldots, N, M, N, N, \ldots\right]$, where $M$ is located in the $n+m$-th position, we have

$$
\begin{gathered}
r(\omega)-2 \varepsilon<r(\beta)<r(\omega)-\varepsilon, \\
\Phi(\beta)>\Phi(\omega),
\end{gathered}
$$

and for any

$$
\gamma=\left[a_{0}, a_{1}, \ldots, a_{n}, N, N, \ldots, N, M, N, \ldots, N, c_{n+m+t+1}, c_{n+m+t+2}, \ldots\right],
$$

$$
\Phi(\gamma)>\Phi(\omega)-2^{-n} .
$$




\section{Admissible Perturbations of Siegel Julia sets}

In the proof of the Main Theorem we will use a sequence of perturbations from last section to "fool" all Turing Machines attempting to compute $J_{\theta}$. In this section we will develop the necessary machinery that would guarantee that the resulting limiting set is locally connected.

Let $N_{0}$ be as in Theorem 4.3, and fix $N>N_{0}$. An admissible irrational number $\theta=$ $\left[a_{1}, a_{2}, \ldots\right] \in \mathbb{T}$ has all of the coefficients $a_{i} \geq N_{0}$, and $a_{j}=N$ for all sufficiently large values of $j$.

One consequence of the renormalization picture we have described above is the following:

Proposition 6.1. There exists $B=B(N)$ such that the following holds. Suppose $\theta$ is an admissible number. Then there exists $k_{0} \in \mathbb{N}$ such that for all $k \geq k_{0}$, denoting $g_{k}$ the cylinder renormalization of $P_{\theta}$ from Theorem 4.2, the boundary of the Siegel disk $\partial \Delta_{g_{k}}$ is a B-quasicircle.

In fact, denoting by $\hat{f}$ the fixed point of $\mathcal{R}_{\mathrm{c} y l}$ with rotation number $[N, N, N, \ldots]$ whose existence is postulated in the Theorem on Hyperbolicity of Renormalization, we see that the boundary of the Siegel disk of $P_{\theta}$ at small scales converges to that of $\hat{f}$ in Hausdorff distance.

Definition 6.1. Now let $\alpha$ be a Brjuno number such that $J_{\alpha}$ is locally connected. We will say that $J_{\beta}$ is an admissible $2^{-n}$-perturbation of $J_{\alpha}$ if the following properties hold.

(1) The Julia set $J_{\beta}$ is locally connected.

$$
\operatorname{dist}_{H}\left(\partial \Delta_{\alpha}, \partial \Delta_{\beta}\right)<2^{-n} \text {. }
$$

(3) For each $n$ and each $\bar{\sigma} \in\{0,1\}^{n}$ denoting $L_{\bar{\sigma}}^{\alpha}$ and $L_{\bar{\sigma}}^{\beta}$ the limbs of $J_{\alpha}$ and $J_{\beta}$ respectively, we have

$$
\left|\operatorname{diam}\left(L_{\bar{\sigma}}^{\alpha}\right)-\operatorname{diam}\left(L_{\bar{\sigma}}^{\beta}\right)\right|<2^{-n}
$$

(4) Consider the Riemann mapping

$$
\Psi_{\alpha}: \mathbb{U} \rightarrow \Delta_{\alpha} \text { normalized by } \Psi_{\alpha}(0)=0, \Psi_{\alpha}^{\prime}(0)=1,
$$

and a similarly defined $\Psi_{\beta}$. Then

$$
\sup _{z \in \overline{\mathbb{U}}}\left|\Psi_{\alpha}-\Psi_{\beta}\right|<2^{-n}
$$

(5) Similarly, denote

$$
\Phi_{\alpha}: \hat{\mathbb{C}} \backslash \overline{\mathbb{U}} \rightarrow \hat{\mathbb{C}} \backslash K_{\alpha}
$$

the Böttcher map of $P_{\alpha}$, and $\Phi_{\beta}$ the Böttcher map of $P_{\beta}$. Let $\gamma_{\alpha}^{ \pm}$be the angles of the two external rays of $J_{\alpha}$, which land at the critical point $p_{\alpha}$, and similarly for $\gamma_{\beta}^{ \pm}$. Then

$$
\left\|\Phi_{\beta}\left(t e^{2 \pi i \gamma_{\beta}^{ \pm}}\right)-\Phi_{\alpha}\left(t e^{2 \pi i \gamma_{\alpha}^{ \pm}}\right)\right\|<2^{-n}
$$

in the spherical norm for $t \in[1, \infty)$. In particular,

$$
\left|p_{\alpha}-p_{\beta}\right|<2^{-n} \text {. }
$$

We have:

Theorem 6.2. Let $\beta_{n}$ be a sequence of Brjuno numbers such that, for each $n, J_{\beta_{n}}$ is a $2^{-n}$ admissible perturbation of $J_{\beta_{n-1}}$. Assume that $\beta_{n} \rightarrow \alpha$, which is another Brjuno number. Then $J_{\alpha}$ is locally connected. 
Proof. By the property (1) of an admissible perturbation and Theorem 2.3 the boundary of $\Delta_{\beta_{n}}$ is a Jordan curve. By the Carathéodory's Theorem, the Riemann mapping $\Psi_{\beta_{n}}$ extends continuously to $S^{1}=\partial \mathbb{U}$. By the properties (2) and (4) of an admissible perturbation, the sequence

$$
\Psi_{\beta_{n}} \underset{\overline{\mathbb{U}}}{\rightrightarrows} \Psi_{\alpha}
$$

Applying the Carathéodory's Theorem to $\Psi_{\alpha}$, we see that $\Delta_{\alpha}$ is a Jordan curve.

By the properties (2) and (5) the critical point $p_{\alpha} \in \partial \Delta_{\alpha}$, and is bi-accessible from infinity. By the property (3), Theorem 2.3, and considerations of continuity the diameters of the limbs of $J_{\alpha}$ of generation $n$ shrink to zero uniformly with $n$.

By Theorem 2.3 the proof is complete.

We now formulate the following key consequence of the result of Inou and Shishikura (cf. the discussion in $[\mathrm{BC} 05])$ :

Proposition 6.3. Consider an admissible number

$$
\alpha=\left[I_{\alpha}, N, N, N, \ldots\right],
$$

where $I_{\alpha}$ is some initial segment of the continued fraction. There exists a Turing Machine which takes as inputs the segment $I_{\alpha}$ and a natural number $\ell$ which outputs $\delta>0$ and $M \in \mathbb{N}$ such that the following holds.

Let $\beta$ be a perturbation of $\alpha$ of the form

$$
\beta=[I_{\alpha}, \underbrace{N, N, \ldots, N}_{m}, A_{1}, A_{2}, \ldots, A_{k}, N, N, N, \ldots], \text { where } m \geq M \text { and } A_{i} \geq N,
$$

and such that

$$
\left|r_{\alpha}-r_{\beta}\right|<\delta
$$

Then

$$
\operatorname{dist}_{H}\left(\partial \Delta_{\alpha}, \partial \Delta_{\beta}\right)<2^{-\ell}
$$

Proof. The boundary of $\Delta_{\alpha}$ is obtained by taking the closure of the critical orbit $\left\{P_{\alpha}^{n}(1)\right\}$. By simple considerations of continuity, there exists $k_{0} \in \mathbb{N}$ such that for every $m \geq k_{0}$,

$$
\partial \Delta_{\alpha} \subset B\left(\partial \Delta_{\beta}, 2^{-\ell}\right) \text {. }
$$

By (3.1) the value of $k_{0}$ can be obtained constructively, given $I_{\alpha}$.

Let $\tau$ be any number larger than $\ell$. For the map $P_{\alpha}$ select $C_{n}$ as in Theorem 4.2, (II). Consider the arc

$$
\ell_{n}=\overline{\partial \Delta_{\alpha} \cap C_{n}}
$$

of the boundary of the Siegel disk trapped inside the fundamental crescent. By the inverse branch $\left(P_{\alpha}\right)^{-1}$, fixing the Siegel disk, it is rotated around the boundary. An inspection shows:

$$
\left(\bigcup_{j=0}^{q_{n}}\left(P_{\alpha}\right)^{-j}\left(\ell_{n-1}\right)\right) \bigcup\left(\bigcup_{j=0}^{q_{n-1}}\left(P_{\alpha}\right)^{-j}\left(\ell_{n}\right)\right) \supset \partial \Delta_{\alpha} .
$$

Denote $W_{n} \subset C_{n}$ the lift of the domain $W$ from Theorem 4.3. Note that by Corollary 4.4,

$$
\ell_{n} \subset \overline{W_{n}} \text {. }
$$


By Theorem 4.2, (II), for any $\nu>0$, we can constructively find $k_{1} \in \mathbb{N}$ such that

$$
W_{n} \Subset B\left(\Delta_{\alpha}, 2^{-\nu}\right)
$$

for $n \geq k_{1}$. An application of Koebe Distortion Theorem to pull-backs

$$
Y_{n} \equiv\left(\bigcup_{j=0}^{q_{n}}\left(P_{\alpha}\right)^{-j}\left(W_{n-1}\right)\right) \bigcup\left(\bigcup_{j=0}^{q_{n-1}}\left(P_{\alpha}\right)^{-j}\left(W_{n}\right)\right)
$$

implies the existence of $k_{2} \in \mathbb{N}$ such that, for $n \geq k_{2}$,

$$
Y_{n} \subset B\left(\Delta_{\alpha}, 2^{-\tau}\right) .
$$

The explicit bound in Koebe Distrotion Theorem again allows us to obtain $k_{2}$ constructively. Set

$$
k=k_{2}+\left|I_{\alpha}\right| .
$$

Now denote $C_{n}^{\prime}, W_{n}^{\prime}$, and $Y_{n}^{\prime}$ the corresponding objects for $P_{\beta}$. By considerations of continuity, for every $d$ we can constructively find

$$
m(d) \gg n \geq k,
$$

such that for $m \geq m(d)$ the domains $C_{n}^{\prime}, C_{n-1}^{\prime}$ are $2^{-d}$-perturbations of $C_{n}, C_{n-1}$ in Hausdorff sense.

By Corollary 4.4, we have

$$
\overline{\Delta_{\beta}} \cap C_{n}^{\prime} \subset W_{n}^{\prime} .
$$

Select $m_{\tau}$ large enough so that for $m>m_{\tau}$ the previous inclusions hold, and

$$
Y_{n}^{\prime} \subset B\left(Y_{n}, 2^{-2 \tau}\right) \text {. }
$$

Then

$$
\overline{\Delta_{\beta}} \subset B\left(\Delta_{\alpha}, 2^{-\tau}\right) .
$$

Thus by moving the perturbation far enough to the right in the continued fraction of $\alpha$, we can guarantee that $\partial \Delta_{\beta}$ does not extend outside a small neighborhood of $\Delta_{\alpha}$.

It remains to ensure, that $\partial \Delta_{\beta}$ does not have decorations which grow deep into $\Delta_{\alpha}$. The easiest way to see this is to note, that by Theorem $3.4, \partial \Delta_{\alpha}$ is a $B$-quasicircle for some explicit $B \in \mathbb{N}$. Hence, for every $\delta>0$, we can constructively find $\tau>2 \ell$ such that setting

$$
U_{\tau}=B\left(\Delta_{\alpha}, 2^{-\tau}\right),
$$

we have

$$
r\left(U_{\tau}, 0\right)-r_{\alpha}<\delta \text {, so that } r\left(U_{\tau}, 0\right)-r_{\beta}<2 \delta .
$$

By Proposition 3.5 applied to uniformization of $U_{\tau}$, we can find $\delta$ small enough, so that

$$
\partial \Delta_{\beta} \subset B\left(\partial U_{\tau}, 2^{-\ell}\right) .
$$

Taking these $\delta$ and $\tau$, and $m>m_{\tau}$ we have

$$
\partial \Delta_{\beta} \subset B\left(\Delta_{\alpha}, 2^{-\ell}\right) \cap B\left(\partial U_{\tau}, 2^{-\ell}\right) \subset B\left(\partial \Delta_{\alpha}, 2^{-\ell}\right) .
$$

We now state: 
Proposition 6.4. Consider an admissible number

$$
\alpha=\left[I_{\alpha}, N, N, N, \ldots\right] \text {. }
$$

There exists a Turing Machine which takes as inputs the segment $I_{\alpha}$ and a natural number $\ell$ which outputs $\delta>0$ and $M \in \mathbb{N}$ such that the following holds.

Let $\beta$ be a perturbation of $\alpha$ of the form

$$
\beta=[I_{\alpha}, \underbrace{N, N, \ldots, N}_{m}, A_{1}, \ldots, A_{k}, N, N, N, \ldots], \text { where } m \geq M \text { and } A_{i} \geq N,
$$

and such that

$$
\left|r_{\alpha}-r_{\beta}\right|<\delta
$$

Then $J_{\beta}$ is an admissible $2^{-\ell}$-perturbation of $J_{\alpha}$.

Proof. Constructively selecting $M$ and $\delta$ to satisfy property (1) is straightforward. Property (2) is proved in Proposition 6.3. Stronger property (4) follows by Proposition 6.3 and Theorem 3.4. and (4)

Properties (3) and (5) follow from an explicit geometric estimate on the size of a limb of $J_{\alpha}$ of generation $n$, given in [Yam99].

\section{Proof of the Main Theorem}

We will now use the machinery developed in Section 6 to prove the Main Theorem. Proof overview. By Theorem 1.1 we know that computability of $r(\theta)$ is equivalent to that of $J_{\theta}$. It is thus sufficient for us to construct a parameter value $\theta$ for which $J_{\theta}$ is locally connected, and no Turing Machine computes $r(\theta)$. We will do this via a diagonalization argument. Let us make a definition:

Definition 7.1. We will say that a parameter $\theta$ fools a Turing Machine $M(n)$ if there exists $m \in \mathbb{N}$ such that on this input $M(m)$ terminates, but does not output a number $r$ with the property

$$
|r-r(\theta)|<2^{-m}
$$

Less formally, the machine $M$ fails to compute $r(\theta)$ by computing a wrong output for some particular choice of the precision parameter $m$.

We construct a parameter $\theta$ that fools all oracle TMs attempting to compute $r(\theta)$. Let us first recall briefly the approach to producing non-computable Julia sets of our paper [BY06]. We would begin by enumerating all oracle Turing Machines $M_{1}^{\phi}, M_{2}^{\phi}, \ldots$ Assuming that a machine in our list computes the conformal radius $r(\theta)$, we can fool it by modifying the parameter $\theta$ far enough in its continued fraction, so that the conformal radius of the Siegel disk decreases by a sufficiently large amount. Applying this idea successively to all of the machines in our list, we end up with a sequence of parameters $\theta_{k}=\left[I_{k}, N, N, \ldots\right]$, such that the finite sequences $I_{1} \subset I_{2} \subset \ldots$ are nested, and such that every machine fails to compute $r\left(\theta_{k}\right)$ starting from some $k$. A passage to the limit, carefully made, produces a parameter with a non-computable Julia set.

Using the bounds of Proposition 6.4, we can ensure that the perturbations are carried out so that the limit is locally connected (see [BY08b]). Turning this argument into a constructive one, however, meets a logical obstacle. When we attempt to fool the machine $M_{k}^{\phi}$, we cannot just "simulate it on $\phi_{k}$, see what it does, and modify the parameter to fool 
it" - the machine $M_{k}^{\phi}$ may not terminate. In fact, we cannot know whether $M_{k}^{\phi}$ terminates without solving the Halting Problem, which is undecidable.

To bypass this difficulty, we run the machines in parallel, and modify the parameter when one of them does output a value $r$. When $M_{k}^{\phi}$ outputs an answer, then we can modify the parameter to fool it. If it never outputs an answer, then it never gets fooled, but this is not a problem, since a machine that never halts cannot be computing $r(\theta)$.

Proof of the Main Theorem. We enumerate all oracle Turing Machines $M_{1}^{\phi}, M_{2}^{\phi}, \ldots$ We will show how to construct a parameter value $\theta$ such that none of the listed machines correctly computes the conformal radius $r(\theta)$.

At every step $k$ of the construction we will maintain a finite initial segment $I_{k}$ of the continued fraction expansion of the final parameter $\theta$, and the current parameter $\theta_{k}=$ $\left[I_{k}, N, N, \ldots\right]$. The segment $I_{k}$ will be an extension of $I_{k-1}$ so that the chain $I_{1} \subset I_{2} \subset \ldots$ converges to the continued fraction expansion of $\theta=\lim _{k \rightarrow \infty} \theta_{k}$.

Also at every step we will maintain a finite status string $S_{k} \in\{0,1\}^{*}$. The status string attaches a status to each machine $M_{j}^{\phi}$ the algorithm is currently considering. $S_{k}[j]=1$ means that $M_{j}^{\phi}$ is fooled by the current parameter $\theta_{k} ; S_{k}[j]=0$ means that it is undetermined, whether $\theta_{k}$ fools $M_{j}^{\phi}$. We also define $S_{k}^{*} \in\{0,1\}^{\omega}$ as the infinite $\{0,1\}$-string obtained from $S_{k}$ by appending an infinite sequence of 0's. The limit $S^{*}$ of the sequence $\left\{S_{k}^{*}\right\}$ is defined as the termwise limit (if one exists). The value $S^{*}[j]$ at the limit will be 1 if the parameter $\theta$ fools $M_{j}^{\phi}$, and $S^{*}[j]$ will be 0 if $M_{j}^{\phi}$ fails to terminate on $\theta$. In either case, $M_{j}^{\phi}$ will fail to compute $r(\theta)$.

We define an order relationship $S_{k} \prec S_{l}$ on the status sequences to be the lexicographical order:

$$
S_{k} \prec S_{l} \text { if } \exists j: S_{k}^{*}[j]=0, S_{l}^{*}[j]=1 \text {, and } \forall(i<j) S_{k}^{*}[i]=S_{l}^{*}[i] .
$$

In our construction, the status strings will satisfy

$$
S_{1} \preceq S_{2} \preceq S_{3} \preceq \ldots
$$

Note that this means, in particular, that for each $j, S_{k}^{*}[j]$ changes its value at most $2^{j}-1$ times as $k$ grows. This implies that the limit

$$
S^{*}=\lim _{k \rightarrow \infty} S_{k}^{*}
$$

exists.

At step $k$ we will be working on fooling the first $k$ machines, thus $\left|S_{k}\right|=k$ for all $k$. For each $j \leq k$ such that $S_{k}[j]=0$ we will maintain a value $\delta_{j}$ which will either be a positive number, or undefined (in which case we will write $\delta_{j}=\perp$ ). This is the amount by which we are willing to drop the conformal radius in order to fool the $j$-th machine. We will further require that

$$
\text { if } i>j \text { then } \delta_{j}>16^{i-j} \delta_{i} \text { (if defined), }
$$

that is, the defined terms in the sequence $\left\{\delta_{j}\right\}$ decrease geometrically. At each step we will also be maintaining an integer $M_{k}$ that specifies beyond which location of the continued fraction expansion we are allowed to change $\theta_{k}$.

We will now describe the initialization and the step in the execution of our construction.

Initialization (iteration $k=0$ ): We set $I_{0}=[N], S_{0}^{*}[j]=0, \delta_{j}=\perp$ for all $j$. 
Step $k \geq 1$ : We start by computing $\delta_{j}$ for $j \leq k$. We compute values of $\delta_{j}$ such that currently $S_{k-1}[j]=0$ and $\delta_{j}=\perp$ (that is, the $j$-th machine has not been fooled, and the value of $\delta_{j}$ is currently undefined). We compute $\delta_{j}>0$ in increasing order of $j, 1 \leq j \leq k$.

We choose $\delta_{j}$ that satisfies $\delta_{j}<16^{-(i-j)} \delta_{i}$ for all $i<j$. We compute a $\delta>0$ and $M^{j}$ as in Proposition 6.4 with $\ell=j$. That is, for each $\beta$ such that

(1) $\beta$ is obtained from $\theta_{k-1}$ by changing finitely many positions $A_{m}$, for $m>M^{j}$, in its continued fraction expansion from $N$ to a bigger number; and

(2) $\left|r\left(\theta_{k-1}\right)-r(\beta)\right|<\delta$,

the set $J_{\beta}$ is an admissible $2^{-j}$-perturbation of $J_{\theta_{k-1}}$. We choose $\delta_{j}<\delta / 4$, we also choose $M_{k}$ such that $M_{k}>2 k$ and $M_{k}>M^{j}$ for all $j \leq k$.

Next, for each $j \leq k$ with $S_{k-1}[j]=0$ we simulate the machine $M_{j}^{\phi}$ on $\theta_{k-1}$ with precision parameter

$$
-\left[\log _{2}\left(\delta_{j} / 8\right)\right]+1
$$

for at most $k$ steps of execution. There are two cases:

Case 1: no machine $M_{i}^{\phi}$ terminates with an answer that is $\delta_{i} / 4$-close to $r\left(\theta_{k-1}\right)$. In this case $I_{k}$ is obtained from $I_{k-1}$ by adding $M_{k}$ digits $N$, thus $\theta_{k}=\theta_{k-1}$ remains unchanged. For each $j$ such that the machine $M_{j}^{\phi}$ terminates (with a wrong answer) we set $S_{k}[j]=1$, for all other $j$ 's we let $S_{k}[j]=S_{k-1}[j]$. The numbers $\delta_{j}$ remain unchanged.

Case 2: at least one machine $M_{i}^{\phi}$ terminates with an answer that is $\delta_{i} / 4$-close to $r\left(\theta_{k-1}\right)$. Let $j$ be the smallest such $i$. We use Lemma 5.3 to compute $I_{k}$ that extends $I_{k-1}$ and has at least $M_{k}$ digits $N$ immediately after $I_{k-1}$ so that

$$
\delta_{j} / 2<r\left(\theta_{k-1}\right)-r\left(\theta_{k}\right)<\delta_{j} \text { and (5.3), (5.4) hold . }
$$

By Lemma 5.3 we can perform the perturbation in such a way that

$$
\Phi\left(\theta_{k-1}\right)<\Phi\left(\theta_{k}\right)<\Phi\left(\theta_{k-1}\right)+C \cdot \delta_{j}
$$

for some constant $C$, as long as $r\left(\theta_{k}\right)$ is bounded away from 0 . We then update $S_{k}[j]$ to 1 and $S_{k}[i]$ for $i>j$ to 0 . We also set $\delta_{i}=\perp$ for $j<i \leq k$.

The intuition behind the last step is that once we have decreased the conformal radius by a large value $\delta_{j}$, all smaller drops intended to fool machines further down the line become irrelevant.

It is evident from the construction that $S_{k-1} \preceq S_{k}$, and hence the $\operatorname{limit} S=\lim S_{k}$ exists. By construction, the sequence $\theta_{k}$ converges to a computable limit $\theta$. We need to see that $r(\theta)$ is non-computable and that $J_{\theta}$ is locally connected.

We first show that $J_{\theta}$ is locally connected. For each $j$ with $S[j]=1$ denote by $k_{j}$ the index of the last iteration when $S[j]$ was set to 1 (recall that there can be at most $2^{j}-1$ such iterations). Then, by the construction, $k_{j}$ is increasing with $j$ and $\theta=\lim _{j \rightarrow \infty} \theta_{k_{j}}$. In addition, by the construction, for each $j, J_{\theta_{k_{j}}}$ is an admissible $2^{-j}$ perturbation of $J_{\theta_{k_{j-1}}}$. Hence by Theorem 6.2 the limit $J_{\theta}$ is locally connected.

We first note that $r(\theta)=\lim r\left(\theta_{k}\right)$. Indeed, it is not difficult to show (see Proposition 5.10 of $[\mathrm{BY} 08 \mathrm{~b}])$ that

$$
r(\theta) \geq r=\lim r\left(\theta_{k}\right)
$$


By (7.2), the values of the Yoccoz-Brjuno function

$$
\infty>\Phi(\theta)>\Phi\left(\theta_{k}\right)-C \cdot\left(r\left(\theta_{k}\right)-r\right), \text { and } \Phi\left(\theta_{k}\right) \geq \Phi\left(\theta_{k-1}\right),
$$

and hence

$$
\Phi(\theta) \geq \lim \Phi\left(\theta_{k}\right)
$$

As the function

$$
v: \theta \mapsto \Phi(\theta)+\log r(\theta)
$$

is continuous, (7.3) and (7.4) imply that $r(\theta)=r$.

Next, we show that $r(\theta)$ is non-computable. Suppose that the machine $M_{k}^{\phi}$ computes $r(\theta)$ for some $k$. There are two cases.

Case 1: $S[k]=1$. This means that for some $\delta$ and $\ell$, an execution of $M_{k}^{\phi}$ on $\theta_{\ell}$ with precision $\delta / 8$ terminates in $\ell$ steps and outputs an answer that is at least $\delta / 4$ away from the correct one. Further changes in $\theta_{\ell}$ happen beyond position $M_{\ell}>2 \ell$ in the continued fraction expansion, and do not affect the execution of $M_{k}^{\phi}$ with precision $\delta / 8$. In addition, by the construction, further changes in $\theta_{\ell}$ will only change the conformal radius by at most $\sum_{i=1}^{\infty} \delta / 16^{i}<\delta / 8$. Thus $M_{k}^{\phi}$ when run on $\theta$ with precision $\delta / 8$ will terminate after $\ell$ steps and output an answer that is at least $\delta / 8$ from $r(\theta)$. Contradiction.

Case 2: $S[k]=0$. In our construction, the value of $\delta_{k}$ is changed finitely many times. Let $\delta$ be the last value of $\delta_{k}$ that is attained after some step $\ell_{1}$. This means that after step $\ell_{1}$ in our construction, no entries $S[j]$ for $j \leq k$ are updated. We claim that $M_{k}^{\phi}$ does not terminate when executed with precision parameter $\delta / 8$ on $\theta$. Suppose for contradiction that it does terminate in $\ell_{2}$ steps. Let $\ell>\max \left(\ell_{1}, \ell_{2}\right)$. Then on iteration $\ell$ of our construction we run $M_{k}^{\phi}$ on $\theta_{\ell-1}$ for $\ell>\ell_{2}$ steps. The execution is identical to the execution of $M_{k}^{\phi}$ on $\theta$ for $\ell$ steps, since $\left|\theta_{\ell-1}-\theta\right|<2^{-\ell-2}$. Hence, by our assumption it must terminate and output a radius value. But if this were the case, our construction would set $S[k]$ to 1 , contradicting the assumption that $S[k]$ is not set to 1 after step $\ell_{1}<\ell$.

Let us make a final observation:

Remark 7.1. Assuming Conjecture 5.2, there is a poly-time algorithm for computing such a parameter $\theta$. In other words, $\theta$ can be computed with precision $2^{-n}$ in time bounded by a polynomial in $n$.

The modifications in the proof to make the algorithm work in polynomial time follow the strategy outlined in $§ 5.2 .3$ of [BY08b] and we will not repeat them here.

\section{Computing $J_{\theta}$ is as hard as solving the Halting Problem}

In this final section we prove that computing the Julia set $J_{\theta}$ constructed in the previous section is as hard as solving the Halting Problem. By Theorem 1.1, the computability of $r(\theta)$ is equivalent to that of $J_{\theta}$. We thus proceed to formulate the following:

Theorem 8.1. Let $\theta$ be the computable parameter from Main Theorem, constructed by the algorithm given in Section $\%$.

(I) There exists and oracle Turing Machine, which, given an oracle for the Halting Problem, computes $r(\theta)$. 
(II) Conversely, there exists an oracle Turing Machine, which, given an oracle for the conformal radius $r(\theta)$, solves the Halting Problem.

Proof of part (I). By Theorem 1.2 the conformal radius $r(\theta)$ is right-computable. Since every right-computable number is computable with an oracle for the Halting Problem, the statement follows.

Proof of part (II). For a TM $M$ denote by $T(M)$ the amount of time $M$ takes to halt. If $M$ does not halt, then set $T(M):=\infty$. We will show that using an oracle for $r(\theta)$ we can compute a function $B(n)$ satisfying the following:

$$
B(n) \geq \max _{M \text { a TM with }|M| \leq n \text { and } T(M)<\infty} T(M) .
$$

In other words, $B(n)$ is a bound on the longest finite amount of time a Turing Machine of description length $n$ can take before halting.

It is clear that if one can compute a function $B(n)$ satisfying (8.1), one can also solve the Halting Problem: on an input $M$ with $|M|=m$ it follows from the definition of $B(m)$ that $M$ will either halt within the first $B(m)$ steps or will never halt. Thus to decide the Halting Problem on $M$ it would suffice to simulate it for $B(m)$ steps and see if it terminates until then.

Denote by $M_{1}$ the machine constructed in the previous section, which produces the parameter $\theta$. Denote by $I_{n}$ the initial segment of $\theta$ the machine $M_{1}$ has generated $n$ steps into the execution. We define a machine $M_{2}$ as follows. The machine $M_{2}$ takes a description of a TM $M$ as an input. It then runs $M$ until it terminates. If $M$ terminates in $K$ steps, the machine $M_{2}$ simulates $M_{1}$ for $K$ iterations to obtain a value

$$
\theta_{K}=\left[I_{K}, N, N, N, \ldots\right] .
$$

$M_{2}(M)$ then outputs $r\left(\theta_{K}\right)$. If $M$ does not halt, then neither does $M_{2}(M)$. The description size of the machine $M_{2}$ is some constant $C_{2}$, so that the total description size of $M_{2}(M)$ is $C_{2}+|M|$.

At each stage of the execution of $M_{1}$, we have the current value of the radius $r\left(\theta_{k}\right)$, as well as the values of the drops $\delta_{j}$ in $r\left(\theta_{k}\right)$ that we will use to fool the machine $M_{j}$ if it hasn't been fooled yet. Using the oracle $\phi$ for the final value of $r(\theta)$ and seeing whether $r(\theta) \approx r\left(\theta_{1}\right)$ or $r(\theta) \approx r\left(\theta_{1}\right)-\delta_{1}$ we can check whether the drop by $\delta_{1}$ has occurred. If it has, we will run $M_{1}$ until the drop occurs, to obtain an updated value for $\delta_{2}$. We will then use the oracle for $r(\theta)$ to check whether a drop by $\delta_{2}$ has occurred. We can continue this process to evaluate the function

$$
D(n):=\text { the number of steps } M_{1} \text { takes before no more }
$$

drops by $\delta_{1}, \ldots, \delta_{n}$ occur.

We claim that

$$
B(n):=D\left(2^{n+C_{2}+1}\right)
$$

satisfies (8.1). The function $B(n)$ obviously can be computed from $D(n)$, and hence it can be computed from an oracle for $r(\theta)$.

Let $M$ be a TM with $|M|=n$. Suppose, for contradiction, that $T(M)>B(n)$. The machine $M_{2}(M)$ can be viewed as an oracle TM $M_{2}(M)^{\phi}$ that ignores its oracle and outputs 
the conformal radius

$$
r_{M}=r\left(\theta_{T(M)}\right)
$$

on all inputs. The description size of $M_{2}(M)^{\phi}$ is bounded by $n+C_{2}$, and hence $M_{2}(M)^{\phi}=$ $M_{L}^{\phi}$, where $L<2^{n+C_{2}+1}$ is the index of $M_{2}(M)^{\phi}$ in the enumeration of all oracle TMs used by $M_{1}$.

By the definition of $D(L)$, we have

$$
\left|r\left(\theta_{k}\right)-r(\theta)\right|<\delta / 4
$$

for all $k \geq D(L)$ where $\delta$ is the current value of $\delta_{L}$ at time $D(L)$. Further, we know that the value of $\delta_{L}$ will not change after time $D(L)$. Equation (8.3) implies that

$$
\left|r_{M}-r\left(\theta_{k}\right)\right|<\delta / 4
$$

for all $k \geq D(L)$. By the assumption we have $T(M)>B(n) \geq D(L)$, thus when $M_{1}$ will simulate $M_{L}^{\phi}$, the simulation will take more than $D(L)$ steps to complete. Hence the first simulation of $M_{L}^{\phi}$ by $M_{1}$ that will terminate will be a simulation with precision $\delta$. By (8.4) output of $M_{L}^{\phi}$ will be consistent with the value of $r\left(\theta_{k}\right)$ within an error of $\delta / 4$. Thus, by the definition of $M_{1}$, it will cause a drop of $\delta$ in the value of $r\left(\theta_{k}\right)$ to fool $M_{L}^{\phi}$. This contradicts (8.4). Hence $B(n) \geq T(M)$, which completes the proof.

\section{REFERENCES}

[BBY07] I. Binder, M. Braverman, and M. Yampolsky, Filled Julia sets with empty interior are computable, Journ. of FoCM 7 (2007), 405-416.

[BC05] X. Buff and A. Chéritat, Ensembles de Julia quadratiques de mesure de Lebesgue strictement positive, Comptes Rendus Math. (2005), no. 341/11, 669-674.

[BC06] - The Brjuno function continuously estimates the size of quadratic Siegel disks, Annals of Math. 164 (2006), no. 1, 265-312.

[BY06] M. Braverman and M. Yampolsky, Non-computable Julia sets, Journ. Amer. Math. Soc. 19 (2006), no. $3,551-578$.

[BY08a] Computability of Julia sets, Moscow Mathematical Journal (2008), Available from http://arxiv.org/abs/math.DS/0610340.

[BY08b] Computability of Julia sets, Springer, 2008.

[dFdM99] E. de Faria and W. de Melo, Rigidity of critical circle mappings I, J. Eur. Math. Soc. (JEMS) 1 (1999), no. 4, 339-392.

[Dou88] A. Douady, Disques de Siegel et anneaux de Herman. Séminaire Bourbaki, Vol. 1986/87, Astérisque 152-153 (1988), no. 4, 151-172.

[Dou93] _ Descriptions of compact sets in c, Topological methods in modern mathematics (Stony Brook, NY, 1991) (1993), 429-465.

[Her85] M. Herman, Are there critical points on the boundaries of singular domains?, Commun. Math. Phys. 99 (1985), no. 4, 593-612.

[Her86] - Conjugaison quasi symétrique des homéomorphismes du cercle à des rotations, and Conjugaison quasi symétrique des difféomorphismes du cercle à des rotations et applications aux disques singuliers de Siegel, available from http://www.math.kyotou.ac.jp/ mitsu/Herman/index.html, 1986.

[IS07] H. Inou and M. Shishikura, Renormalization for parabolic fixed points and their perturbations, Preprint (2007).

[Lyu86] M Lyubich, Dynamics of rational transformations: topological picture, Russian Math. Surveys 41 (1986), no. 4, 43-117.

[MMY97] S. Marmi, P. Moussa, and J.-C. Yoccoz, The Brjuno functions and their regularity properties, Commun. Math. Phys. 186 (1997), 265-293. 
[Pet96] C. Petersen, Local connectivity of some Julia sets containing a circle with an irrational rotation, Acta Math. 177 (1996), 163-224.

[PZ04] C. Petersen and S. Zakeri, On the Julia set of a typical quadratic polynomial with a Siegel disk, Ann. of Math. 159 (2004), no. 1, 1-52.

[Sul83] D. Sullivan, Conformal dynamical systems, Geometric Dynamics (Palis, ed.), Lecture Notes Math., vol. 1007, Springer-Verlag, 1983, pp. 725-752.

[Thu] W Thurston, On the combinatorics and dynamics of iterated rational maps, Preprint.

[Wei00] K. Weihrauch, Computable Analysis, Springer-Verlag, Berlin, 2000.

[Yam99] M. Yampolsky, Complex bounds for renormalization of critical circle maps, Erg. Th. \& Dyn. Systems 19 (1999), 227-257.

[Yam02] M Yampolsky, Hyperbolicity of renormalization of critical circle maps, Publ. Math. Inst. Hautes Études Sci. 96 (2002), 1-41.

[Yam08]__ Siegel disks and renormalization fixed points, Fields Institute Communications 53 (2008).

[Yoc95] J.-C. Yoccoz, Petits diviseurs en dimension 1, S.M.F., Astérisque 231 (1995).

[YZ01] M. Yampolsky and S. Zakeri, Mating Siegel quadratic polynomials, J. Amer. Math. Soc. 14 (2001), no. $1,25-78$. 